MATEC Web of Conferences 40, 02004 (2016)

DOI: $10.1051 /$ matecconf/20164002004

(c) Owned by the authors, published by EDP Sciences, 2016

\title{
Development of Exoskeletons and Applications on Rehabilitation
}

\author{
Xinyu Guan, Linhong Ji and Rencheng Wang \\ Division of Intelligent and Bio-mimetic Machinery, The State Key Laboratory of Tribology, Tsinghua University, China
}

\begin{abstract}
For over a century, the development of exoskeletons experienced five periods including sprout, exploration, dormancy, accumulation and climax period from a concept in $19^{\text {th }}$ century to applications in distinctive fields in $21^{\text {th }}$ century. Recently, exoskeletons are applied in military, civilian and rehabilitation to augment the travel and loading abilities of soldiers, increase an operator's load-handling capabilities, reduce the occurrence of musculoskeletal disorders, and improve the lost functions and quality of life of patients, respectively. Aiming at lessening the strain on physical therapists to train patients with severe or degenerative disabilities, motor cognitive limitation and improving their quality of life, exoskeletons are applied on the field of rehabilitation, mainly on patient training and locomotion. Although great progress has been made in the century long effort to design and implement exoskeletons, many design challenges still remain including powered devices, the comfort of human-machine interface and how to effectively understand the wearer's intensions.
\end{abstract}

\section{Introduction}

The term 'exoskeleton' was used in biology referring to the chitinous or calcified external skeleton used by numerous animal taxa for structural support and defense against predators [1]. Now, the exoskeletons are generally regarded as a technology that extends, complements, substitutes or enhances human function and capability or empowers the human limb where it is worn. Different from other robots, the operator of an exoskeletons is human who need to make decisions [2] and perform tasks with exoskeletons. Through combining human intelligence and machine power exoskeletons enhance the abilities of both human power and machine intelligence [3].

Since the concept of exoskeleton was produced in the $19^{\text {th }}$ century, the development of exoskeletons have undergone five phases, i.e. sprout period, exploration period, dormancy period, accumulation period and climax period $[4,5]$. Exoskeletons apply and merge manifold techniques involving mechanical and electronic engineering, automation technology, biological, medical, and material science [3]. Recently, exoskeletons are applied in military, civilian and rehabilitation [4]. For military purposes, the exoskeletons are designed to augment the travel and loading abilities of soldiers [6]; for civil applications, the exoskeletons are used to increase an operator's load-handling capabilities and reduce the occurrence of musculoskeletal disorders, or for rescue; for rehabilitation, exoskeletons are aiming at improving the lost functions $[6,7]$ and the quality of life of patients with severe or degenerative disabilities, motor cognitive limitation [8].

\section{Development of exoskeletons}

\subsection{Sprout period}

The sprout period lasted more than one century from 1830 to 1960 . During this period, (1) A British inventor Robert Seymour proposed the concept to help people walk by a wearable device which was propelled by steam in 1830; (2) An American inventor Ira C. C. Rinehart conceptually designed a walking machine which enabled an individual to step seven feet and four inches at an ordinary stride in 1889; (3) From 1889 to 1890 , Nicholas Yagn, of St.Petersburg, Russia, designed a walking, jumping, and running assisted device using a giant leaf spring; (4) In 1890, another inventor Yagn designed an exoskeleton with long leaf springs in parallel to the legs to help people run faster and jump higher. In stance phase, the weight of body can be transferred to the ground directly by the spring to reduce the forces on the standing leg. Most exoskeletons were conceptual design in sprout period due to the limitations of the technology at that time.
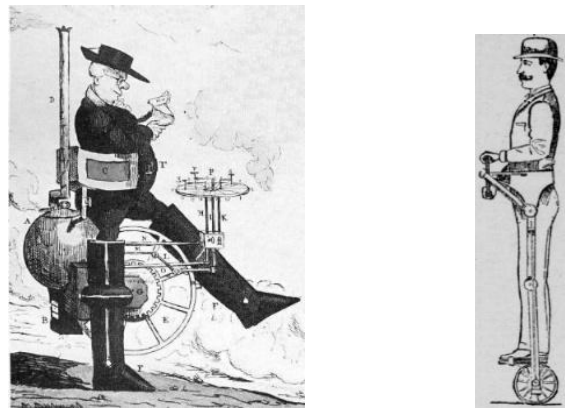
(a)

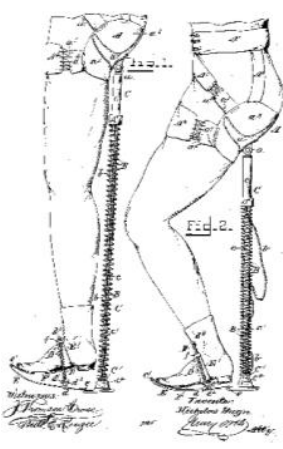

(c) (b)

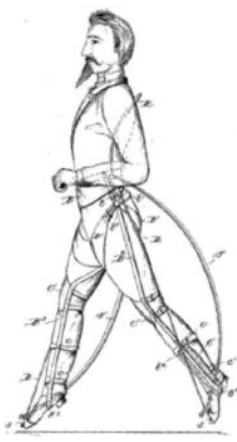

(d)
Figure 1. Exoskeletons in sprout period: (a) exoskeleton designed by Robert Seymour; (b) exoskeleton designed by Ira C. C. Rinehart; (c) exoskeleton designed by Nicholas Yagn; (d) exoskeleton designed by Yagn

\subsection{Exploration period}

The exoskeleton HARDIMAN developed by the US Department and General Electric in 1965 marked the exoskeleton development entering the exploration period. HARDIMAN aimed at augmentation that the individual who worn it could lift $1,500 \mathrm{lbs}(682 \mathrm{~kg})$ [4]. In fact, only one arm of HARDIMAN was developed and achieved to lift $750 \mathrm{lbs}(341 \mathrm{~kg})$ until the $1970 \mathrm{~s}$. The failure of HARDIMAN was mainly caused by which the energy supplies were too huge to be portable, and the speed of data processing and function control was slow [4]. In the late $1960 \mathrm{~s}$ and $1970 \mathrm{~s}$, an active anthropomorphic exoskeletons with pneumatic power and partly kinematical program for paraplegics was developed at the Mihailo Pupin Institute under the Prof. Vukobratovic's guidance. At the same time, the theory of legged locomotion systems was first put forward by Prof. Vukobratovic, which established foundation for present modern high-performance exoskeletons[9]. The researchers of University of Wisconsin started to develop a full lower limb exoskeleton in 1968 [10]. This exoskeleton was designed to help those paraplegics with complete upper limb capabilities to walk again. The wearer can implement the sit-to-stand, stand-to-sit translation and walk at $50 \%$ of normal speed. The hip and ankle joint both had three rotational degrees of freedom (DOF) and the knee joint had one rotational DOF. The joints at hip and knee for flexion/extension were actuated by hydraulic power, and the other joints were passive [11]. Although this exoskeleton was developed for paraplegics, there was not any report about the relevant tests [6].

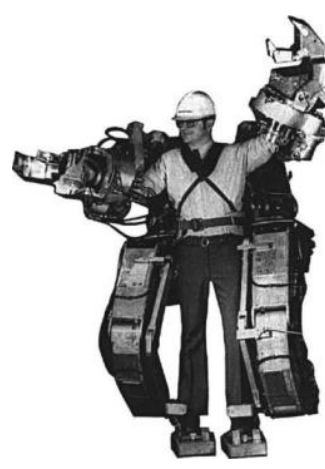

(a)

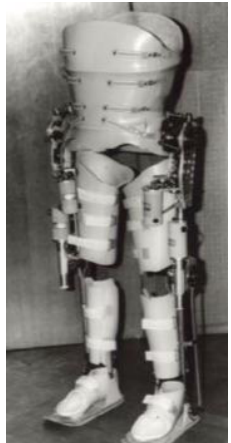

(b)

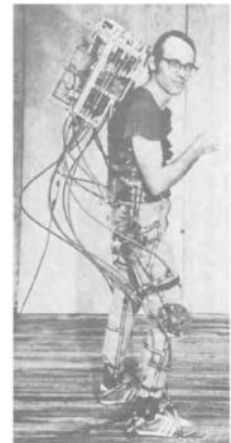

(c)
Figure 2. Exoskeletons in exploration period: (a) HARDIMAN by General Electric; (b) active anthropomorphic exoskeleton by Mihailo Pupin Institute; (c) lower limb exoskeleton by

University of Wisconsin

\subsection{Dormancy period}

The development of exoskeleton entered the dormancy period in the 1980s. In the middle 1980s, the exoskeleton concept "Pitman" was put forward by Jeffrey Moore at the Los Alamos National Laboratory (Los Alamos, NM) to apply in military to augment the soldiers' capabilities. However, this exoskeleton program was not funded by the U.S. Defense Advanced Research Projects Agency (DARPA). In 1988, Prof. Jichuan Zhang started to research the electric walking machine for high leg paraplegia patients at Tsinghua University. Using bar linkage mechanism, the ipsilateral hip joint and knee joint of the exoskeleton were actuated by only one motor. This structure decreased the weight of the exoskeleton and became more compact and portable. In 1990, G. John Dick and Eric A. Edwards developed SpringWalker according to the mechanism that a device in series with the human leg can reduce the metabolic cost of running by lowering impact losses and by providing energy return [12]. However, SpringWalker can only enhance jumping height. For running, it even increased metabolic cost by $20 \%$ compared to locomotion without it [5].

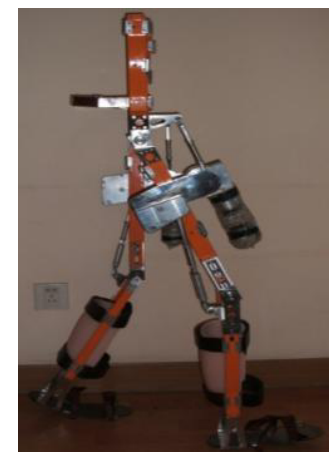

(a)

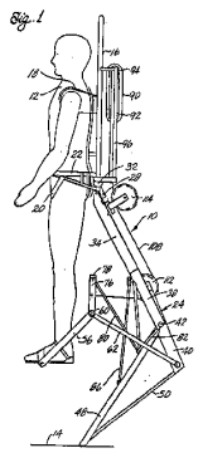

(b)
Figure 3. Exoskeletons in dormancy period: (a) Electric walking machine for high leg paraplegia patients of Tsinghua University; (b) SpringWalker

\subsection{Accumulation period}


From 1990 to 2000, the research of exoskeleton went into the accumulation period. In 1992, Prof. Yoshiyuki Sankai of University of Tsukuba started to develop a wearabletype robot 'Robot Suit HAL' (Hybrid Assistive Limb), which was intended to physically support a wearer's daily activities and heavy work [13]. The first prototype named HAL-1 adopted DC motors and ball screws to augment the wearer's joint torque [14]. In 1994, researchers of Kanagawa Institute of Technology developed a wearable power assisting suit for nurses to enhance their muscle strength to lift patients and avoid back injuries [15]. The movement of the joints at arms, waist and legs of the suit were sensed by strain sensors to detect the muscle force and actuated by pneumatic rotary actuators with concentric round boxes sliding each other [16, 17]. Compared to the over-ground exoskeletons, Hocoma AG developed an immobile exoskeleton Lokomat consisting of an over-ground exoskeleton, an advanced body weight support system (BWS) and a treadmill in 2000 at Switzerland [18, 19]. The Lokomat with repetitive walking on one hand help to improve circulation, strengthen bones and muscles and gain a natural walking pattern [20], on the other hand decrease the physical effort and constraint of the therapists [19].

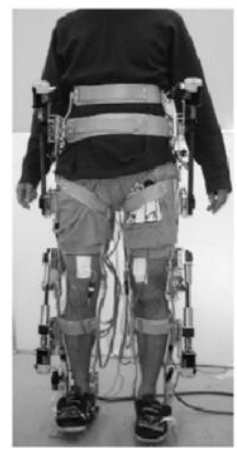

(a)

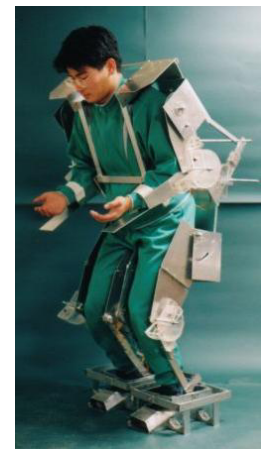

(b)

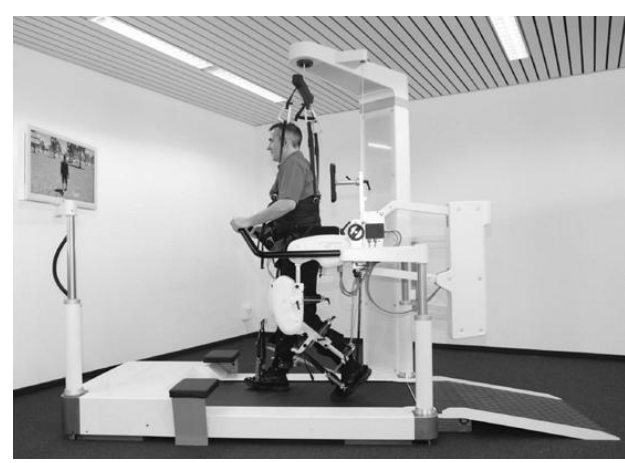

(c)

Figure 4. Exoskeletons in accumulation period: (a) HAL-1 of University of Tsukuba; (b) suit sliding boxes actuator of Kanagawa Institute of Technology; (c) Lokomat of Hocoma AG

\subsection{Climax period}

Exoskeletons attracted much more attention of researchers from different countries including US, Japan, Israel, France, Switzerland, South Korea, China, etc. and the development of exoskeletons went into the climax period since 2000 .

One representative of exoskeletons applied in military, Berkeley Lower Extremity Exoskeleton (BLEEX) was developed to increase soldier's load capacity, lessen the risk of leg and back injury, decrease the metabolic consumption and reduce the perceived level of difficulty [5]. BLEEX adopted the hybrid hydraulic-electric portable power supply [21] in order to achieve carrying its own power source [22]. The hip and ankle joint of BLEEX had three DOFs, respectively, among which hip flexion/extension, abduction/adduction and ankle flexion/extension were actuated by linear hydraulic actuators. Its knee joint had one DOF actuated for flexion/extension [23]. The control system of BLEEX mainly collected sensory information from exoskeletons to determine the kinematic and dynamic parameters [23]. It was reported that the soldier who wore BLEEX can walk at $0.9 \mathrm{~m} / \mathrm{s}$ with load up to $75 \mathrm{~kg}$ and $1.3 \mathrm{~m} / \mathrm{s}$ without load [6].

The representative civil application of exoskeletons was the Robot Suit Hybrid Assistive Limb (HAL)-5 developed by Professor Yoshiyuki Sankai at University of Tsukuba for both power augmentation and walk assistance [24, 25]. The hip and ankle joint of HAL-5 were actuated by a DC motor with harmonic drive for flexion/extension, respectively, and the ankle joint for flexion/extension DOF was passive with springs to return a normal angle [26]. HAL-5 adopted joint torque augmentation at the hip, knee and ankle joint, which is different from BLEEX transferring the load to ground. HAL-5 had two types of control systems: "Cybernic Voluntary Control System" and "Cybernic Autonomous Control System" [26]. Cybernic Voluntary Control System understood the wearer's voluntary intention according to the surface electromyographic (sEMG) signals through placing the sEMG electrodes below the hip and above the knee [5]. Then the power units of HAL-5 generated power assist torque by amplifying the wearer's joint torque estimated from sEMG signals [26]. Cybernic Autonomous Control System was developed to provide effective physical supports for the handicaps by the potentiometers, ground reaction force sensors, a gyroscope and accelerometer on the backpack to estimate the posture since the signals of handicaps could cause a broken walking pattern $[1,5,26]$.

ReWalk from Argo Medical Technologies has been commercialized for fundamentally changing the health and life experiences of individuals with spinal cord injuries (SCI). It consisted of a wearable brace support suit with DC motors at hip and knee joint, respectively, rechargeable batteries, a computer-based controller contained in a backpack, a wireless mode selector, and an array of sensors that measure upper-body tilt angle, joint angles, and ground contact [27]. ReWalk utilized a closed-loop algorithm software control and triggered and maintained the walking pattern by detecting the wearer's upper-body movements. Additionally, ReWalk can also help the wearer climb stairs, transform from sitting to standing and vice versa. The crutches were necessary to keep balance. 


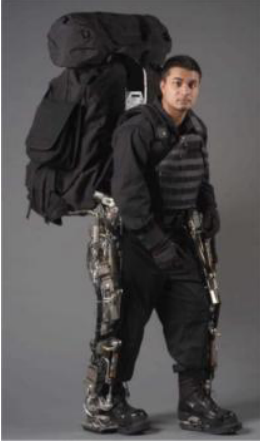

(a)

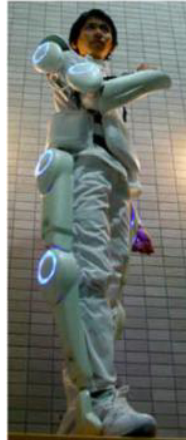

(b)

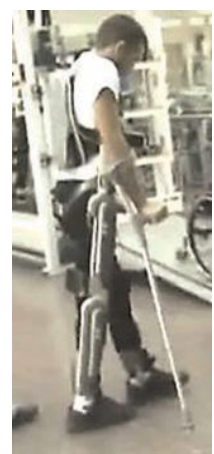

(c)
Figure 5. Exoskeletons in climax period: (a) BLEEX; (b) HAL5; (c) ReWalk

\section{Rehabilitation applications}

Rehabilitation exoskeletons are usually applied on two aspects. One can provide greater repeatability and lessen the strain on physical therapists to train stroke survivors. The other is to help the patients with spinal cord injury or muscle atrophy walk and improve their activities of daily living (ADL).

Lower-limb exoskeletons for rehabiliation were grouped as treadmill gait trainers, foot-plate-based gait trains, overground gait trainers and stationary gait trainers according to rehabilitation principle (Fig. 6) [28]. Treadmill gait trainer system combines BWS and exoskeleton type robots. Foot- plate-based gait trains system achieves to simulate different gait patterns through controlling the movement trajectory of the separate foot plates on which patients' feet position on. Overground gait trainers system allow patients move under their own control rather than moving them through predetermined movement patterns. Stationary gait trainers system aims at obtaining effcient strengthening of the muscles, endurance development, joint mobility and movement coordination.

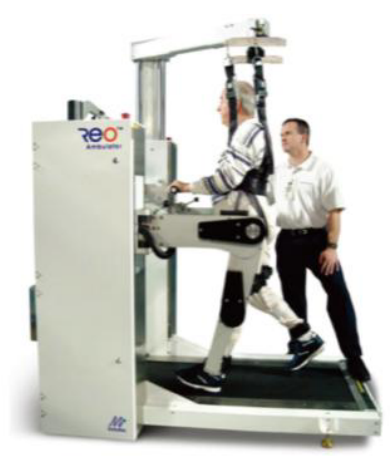

(a) (b)

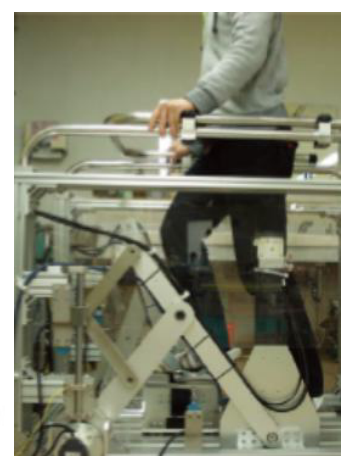

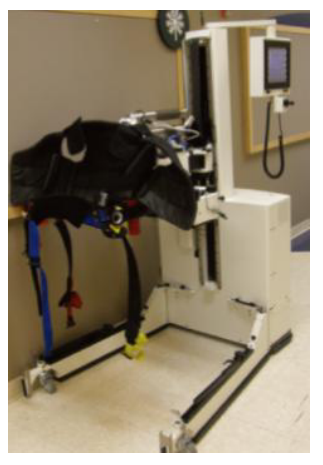

(c)

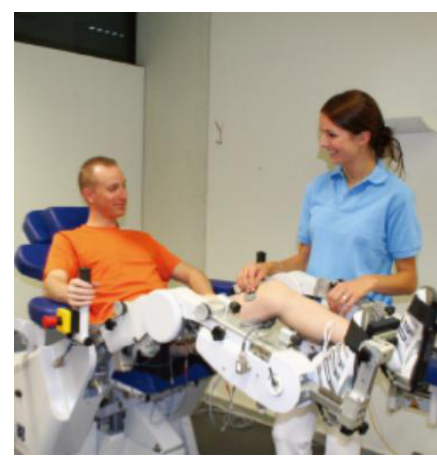

(d)
Figure 6. Lower-limb rehabilitation exoskeletons for training: (a) treadmill gait trainers; (b) foot-plate-based gait trains; (c) overground gait trainers; (d) stationary gait trainers

Exoskeletons for locomotion can be divided into openchain structure and coupling structure based on mechanical structure. The open-chain structure is that the exoskeleton joints are separately actuated while the coupling structure refers to the structure that one actuator simultaneously actuates more than one joint coupled by links or cables. The exoskeleton developed by Tsinghua University (Fig. 3a) used coupling structure which has advantages of light weight and less power. However, most exoskeletons (e.g. HAL-5, ReWalk, etc.) adopted the open-chain structure $[18,23,26,29]$ as this structure is designed easily and controlled simply compared to the coupling structure. Additionally, this structure can be redesigned or extended conveniently based on the original level. The movements of exoskeleton joints with open-chain structure are coordinated through the control system.

\section{Challenges}

Although great progress has been made in the century long effort to design and implement exoskeletons, many design challenges still remain.

The first challenge is the power of exoskeleton. Heavy powered devices limit torque and power. Therefore, developing light power system and small actuators are necessary. The second challenge is the human-machine interface. The interface designs often cause discomfort when wearing the exoskeletons, which limit the length of time that a device can be worn. It is certainly an achievable goal to combine the exoskeletons and the human body harmoniously and effectively to guarantee comfort. The third challenge is how to understand the intension of wearers, especially the patients with SCI who lost neurotransmission below injured spinal canal. Although rehabilitation exoskeletons like HAL-5 collect and analyze surface electromyography (sEMG) signal to detect the wearer's intension, there are less direct information exchange between current exoskeletons and wearers' nervous system. Thus it is critically important to develop neural technology on human-machine direct information exchange. 


\section{Conclusions}

In this paper, an insight review has been conducted on the development of exoskeletons, their applications on rehabilitation and current challenges. The development of exoskeletons experienced five periods including sprout, exploration, dormancy, accumulation period and climax period. Especially in the climax period, more and more rehabilitation exoskeletons were developed to lessen the strain on physical therapists to train or assist patients. Although great progress has been made in the century long effort to develop exoskeletons, many design challenges still remain.

\section{References}

1. E. Guizzo and H. Goldstein, Spectrum, IEEE 42, 50-56 (2005).

2. J. M. P. Gunasekara, R. A. R. C. Gopura, T. S. S. Jayawardane, and S. W. H. M. T. Lalitharathne, in System Integration (SII), 19-24 (2012).

3. C. J. Yang, J. F. Zhang, Y. Chen, Y. M. Dong, and Y. Zhang, Proceedings of The Institution of Mechanical Engineers Part C-journal of Mechanical Engineering Science 222, 1599-1612 (2008).

4. R. Bogue, Industrial Robot: An International Journal 36, 421-427 (2009).

5. H. Herr, J NeuroEngineering Rehabil 6, 1-9 (2009).

6. A. M. Dollar and H. Herr, Robotics, IEEE Transactions on 24, 144-158 (2008).

7. F. Yakub, A. Z. Md Khudzari, and Y. Mori, International journal of rehabilitation research. Internationale Zeitschrift fur Rehabilitationsforschung. Revue internationale de recherches de readaptation 37, 9-21 (2014).

8. T. Saito, T. Shibata, K. Wada, and K. Tanie, in Computational Intelligence in Robotics and Automation, 2003.1, 119-124 (2003).

9. M. K. Vukobratovic, International Journal of Humanoid Robotics 4, 459-486 (2007).

10. J. Grundmann and A. Seireg, Theory Pract. Robots Manipulators, 233-240 (1977).

11. A. Seireg and J. G. Grundmann, Biomechanics of Medical Devices, 569-644 (1981).

12. T. A. McMahon and P. R. Greene, Journal of biomechanics 12, 893-904 (1979).

13. Y. Sankai, in Robotics Research, 25-34 (2011).

14. F. Ichihashi, Y. Sankai, and S. Kuno, International Journal of Sport and Health Science 4, 617-627 (2006).

15. K. Yamamoto, M. Ishii, H. Noborisaka, and K. Hyodo, in Robot and Human Interactive Communication, 2004. 661-666, (2004).

16. K. Yamamoto, K. Hyodo, and T. Matsuo, in Proceedings of the JFPS International Symposium on Fluid Power, 415-420, (1996).

17. K. Yamamoto, K. Hyodo, M. Ishii, and T. Matsuo, JSME International Journal Series C Mechanical
Systems, Machine Elements and Manufacturing 45, 703-711 (2002).

18. G. Colombo, M. Joerg, R. Schreier, and V. Dietz, J Rehabil Res Dev 37, 693-700 (2000).

19. R. Riener, L. Lunenburger, S. Jezernik, M. Anderschitz, G. Colombo, and V. Dietz, IEEE transactions on neural systems and rehabilitation engineering 13, 380-94 (2005).

20. M. Wellner, T. Thuring, E. Smajic, J. von Zitzewitz, A. Duschau-Wicke, and R. Riener, Studies in health technology and informatics 125, 497-9 (2007).

21. K. Amundson, J. Raade, N. Harding, and H. Kazerooni, in Intelligent Robots and Systems, 2005., 3453-3458 (2005).

22. H. Kazerooni and R. Steger, Journal of dynamic systems, measurement, and control 128, 14-25 (2006).

23. A. B. Zoss, H. Kazerooni, and A. Chu, Mechatronics 11, 128-138 (2006).

24. H. Kawamoto and Y. Sankai, in Computers Helping People with Special Needs. 2398, 196-203(2002).

25. H. Kawamoto, L. Suwoong, S. Kanbe, and Y. Sankai, in Systems, Man and Cybernetics, 2003. 2, 16481653 (2003).

26. Y. Sankai, in Robotics Research. 66, 25-34 (2011).

27. G. Zeilig, H. Weingarden, M. Zwecker, I. Dudkiewicz, A. Bloch, and A. Esquenazi, J Spinal Cord Med 35, 96-101 (2012).

28. I. Díaz, J. J. Gil, and E. Sánchez, Journal of Robotics, 2011 (2011).

29. A. Esquenazi, M. Talaty, A. Packel, and M. Saulino, American Journal of Physical Medicine \& Rehabilitation, 91, 911-921 (2012). 